\title{
NEW RESULTS ON THE PHASE DIAGRAM OF THE FFXY MODEL: A TWISTED CFT APPROACH
}

\author{
G. CRISTOFANO and V. MAROTTA \\ Dipartimento di Scienze Fisiche and INFN, Università di Napoli "Federico II", \\ Via Cintia, Compl. Universitario M. Sant'Angelo, 80126 Napoli, Italy \\ E-mail: cristofano@na.infn.it \\ P. MINNHAGEN \\ Department of Theoretical Physics, Umea University, \\ 90187 Umea, Sweden \\ E-mail: Petter.Minnhagen@physics.umu.se \\ A. NADDEO \\ CNISM, Unit $\mu$ a di Ricerca di Salerno, \\ Via Salvador Allende, 84081 Baronissi (SA), Italy \\ E-mail: naddeo@sa.infn.it \\ G. NICCOLI \\ LPTM, Université de Cergy-Pontoise, \\ 2 avenue Adolphe Chauvin, 95302 Cergy-Pontoise, France \\ E-mail: Giuliano.Niccoli@ens-lyon.fr
}

\begin{abstract}
The issue of the number, nature and sequence of phase transitions in the fully frustrated $X Y$ (FFXY) model is a highly non trivial one due to the complex interplay between its continuous and discrete degrees of freedom. In this contribution we attack such a problem by means of a twisted conformal field theory (CFT) approach [1] and show how it gives rise to the $U(1) \otimes Z_{2}$ symmetry and to the whole spectrum of excitations of the FFXY model [2].
\end{abstract}




\section{Introduction: the state of art}

The phase diagram of the FFXY model has been the subject of intensive studies in the last thirty years, due to the presence of the mixed symmetry $U(1) \otimes Z_{2}$. But a full and definitive answer to such a question still lacks; in this paper we address the problem by means of a twisted CFT approach [1]. The XY model on a square lattice in the presence of an external magnetic field transversal to the lattice plane is described by the action:

$$
H=-\frac{J}{k T} \sum_{\langle i j\rangle} \cos \left(\varphi_{i}-\varphi_{j}-A_{i j}\right),
$$

where $\{\varphi\}$ are the phase variables on the sites, the sum is over nearest neighbors, $J>0$ is the coupling constant and the bond variables $A_{i j}=\frac{2 e}{\hbar c} \int_{i}^{j} A \cdot d l$ satisfy the full frustration condition $\sum_{\text {plaquette }} A_{i j}=\pi$. Choosing the Landau gauge in such a way to get a lattice where each plaquette displays one antiferromagnetic and three ferromagnetic bonds, we obtain two ground states with opposite chiralities. The discrete $Z_{2}$ symmetry of the FFXY model is broken at low temperature and will be restored beyond a certain temperature after the formation of domain walls separating islands of opposite chirality. The Ising transition overlaps to a vortex-unbinding transition, which is associated with the continuous $U(1)$ symmetry [3]. The action (1) can be rewritten as a fractionally charged Coulomb gas (CG) defined on the dual lattice, $H_{C G}=-\frac{J}{k T} \sum_{r, r^{\prime}}\left(m(r)+\frac{1}{2}\right) G\left(r, r^{\prime}\right)\left(m\left(r^{\prime}\right)+\frac{1}{2}\right)$, where $\lim _{\left|r-r^{\prime}\right| \rightarrow \infty} G\left(r, r^{\prime}\right)=\log \left|r-r^{\prime}\right|+\frac{1}{2} \pi$ and the neutrality condition $\sum_{r}\left(m(r)+\frac{1}{2}\right)=0$ holds. Such a model exhibits two possible phase transitions, an Ising and a vortex-unbinding one. The issue whether there are two distinct phase transitions, $T_{V}>T_{d w}$ or $T_{V}<T_{d w}$ with $T_{V}$ and $T_{d w}$ marking respectively the breaking of $U(1)$ and of $Z_{2}$ symmetry [4], or a single transition with the simultaneous breaking of both symmetries [5] has been widely investigated. The model allows for the existence of two topological excitations: vortices and domain walls. Vortices are point-like defects such that the phase rotates by $\pm 2 \pi$ in going around them [3]. A domain wall can be viewed as a line on the square lattice, each segment of which separates two cells with the same chirality [6]. If the domain walls form a right angle, such corners must behave as fractional vortices with topological charge $\pm 1 / 4$

[3]. There exists a temperature $T_{d w}$ such that, when $T>T_{d w}$, dissociation of 
bound pairs of fractional vortices is allowed, which triggers the dissociation of pairs of ordinary vortices. The system undergoes two phase transitions with temperatures such that $T_{V}<T_{d w}$ [7]. At finite temperatures kinks may appear on the domain wall. Simple kinks must behave as fractional vortices with topological charge $\pm 1 / 2$ while a double kink does not introduce mismatches in the phase distribution. At low temperatures, all simple kinks are bound into neutral pairs. As the temperature increases, a phase transition in the gas of logarithmically interacting kinks leads to pair dissociation and emergence of free simple kinks [6]. That takes place at $T_{K}<T_{V}$ [8] and produces two distinct bulk transitions with $T_{V}<T_{d w}$. A more general conclusion is reached by studying the coupled XY-Ising model $[9,10]$, which is in the same universality class of the FFXY model. Such a model can be introduced starting with a system of two XY models coupled through a symmetry breaking term [9]:

$$
H=A\left[\sum_{i=1,2} \sum_{\left\langle r, r^{\prime}\right\rangle} \cos \left(\varphi^{(i)}(r)-\varphi^{(i)}\left(r^{\prime}\right)\right)\right]+h \sum_{r} \cos 2\left(\varphi^{(1)}(r)-\varphi^{(2)}(r)\right) \text {. }
$$

The limit $h \rightarrow 0$ corresponds to a full decoupling of the fields $\varphi^{(i)}, i=$ 1,2 , while the $h \rightarrow \infty$ limit corresponds to the phase locking $\varphi^{(1)}(r)-$ $\varphi^{(2)}(r)=\pi j, j=1,2$; as a consequence the model gains a symmetry $U(1) \otimes Z_{2}$ and its Hamiltonian renormalizes towards the XY-Ising model [9]. Its phase diagram $[9,10,12]$ is built up with three branches which meet at a multicritical point $P$. Two branches describe separate Kosterlitz-Thouless (KT) and Ising transitions while the third $(P T)$ corresponds to single transitions with simultaneous loss of XY and Ising order. It becomes a first order one at a tricritical point $T$ and seems to be non-universal [9]; in fact the numerical estimate for the central charge, $c \sim 1.60$, is higher than the value $c=3 / 2$, pertinent to a superposition of critical Ising and gaussian models [11]. Indeed the central charge seems to vary continuously from $c \approx 1.5$ near $P$ to $c \approx 2$ at $T$ [10]. The system lacks conformal invariance [9], so one could consider suitable perturbations of the XY-Ising model as a starting point to study the vicinity of the point $P[12]$. Instead, recent numerical simulations on huge lattices [13] lead to two very close but separate transitions on the $P T$ line. A possible solution could be [7] the addition of an antiferromagnetic coupling $(\bar{J})$ term to the Coulomb gas Hamiltonian $H_{C G}$; for $\bar{J} \neq 0$, the two 
transitions on the PT line separate with the KT one occurring at a lower temperature [7]. On the other side, by adding higher harmonics contributions to the potential $[11,14]$, the possibility of a merging critical point $T$ will be provided here in the context of a twisted CFT approach [1], which extends the results of Ref. [11], so recovering the whole phase diagram [9, 10, 12].

\section{2 m-reduction procedure}

In this Section we recall those aspects of the twisted model (TM) which are relevant for the FFXY model. We focus in particular on the $m$-reduction procedure for the special $m=2$ case [1], since we are interested in a system with $U(1) \otimes Z_{2}$ symmetry. Such a theory describes well a system consisting of two parallel layers of $2 \mathrm{D}$ electron gas in a strong perpendicular magnetic field, with filling factor $\nu^{(a)}=\frac{1}{2 p+2}$ for each of the two $a=1,2$ layers [1]. Regarding the integer $p$, characterizing the flux attached to the particles, we choose the "bosonic" value $p=0$, since it enables us to describe the highly correlated system of vortices with flux quanta $\frac{h c}{2 e}$. Let us start from the "filling" $\nu=\frac{1}{2}$, described by a CFT with $c=1$ in terms of a scalar chiral field $Q(z)=q-i p \ln z+i \sum_{n \neq 0} \frac{a_{n}}{n} z^{-n}$, compactified on a circle with radius $R^{2}=$ $1 / \nu=2$; here $a_{n}, q$ and $p$ satisfy the commutation relations $\left[a_{n}, a_{n^{\prime}}\right]=n \delta_{n, n^{\prime}}$ and $[q, p]=i$. From such a CFT (mother theory), using the $m$-reduction procedure, which consists in considering the subalgebra generated only by the modes in $Q(z)$ which are a multiple of an integer $m$, we get a $c=m$ orbifold CFT (the TM), which is symmetric under a discrete $Z_{m}$ group and, for $m=2$, will be shown to describe the whole phase diagram of the FFXY model. Its primary fields content, for the special $m=2$ case, can be expressed in terms of two scalar fields given by:

$$
X(z)=\frac{1}{2}(Q(z)+Q(-z)), \phi(z)=\frac{1}{2}(Q(z)-Q(-z))
$$

$X(z)$ is $Z_{2}$-invariant and describes the electrically "charged" sector of the new theory, while $\phi(z)$ satisfies the twisted boundary conditions $\phi\left(e^{i \pi} z\right)=$ $-\phi(z)$ and describes the "neutral" sector [1]. The TM primary fields are composite vertex operators $V(z)=U_{X}(z) \psi(z)$, where $U_{X}(z)$ is the vertex describing its "charge" content and $\psi(z)$ describing the "neutral" one. In the neutral sector it is useful to introduce the two chiral operators $\psi(z)(\bar{\psi}(z))=$ 
$\frac{1}{2 \sqrt{z}}\left(: e^{i \alpha \phi(z)}: \pm: e^{i \alpha \phi(-z)}:\right)$, with only the first one obeying the boundary conditions. In a fermionized theory they correspond to two $c=1 / 2$ Majorana fermions with Ramond and Neveu-Schwartz boundary conditions [1] and, in the TM, they appear to be not completely equivalent. In fact the whole TM theory decomposes into a tensor product of two CFTs, a $Z_{2}$ invariant one with $c=3 / 2$ and symmetry $U(1) \otimes Z_{2}$ and the remaining $c=1 / 2$ one realized by a Majorana fermion in the twisted sector. Furthermore the energy-momentum tensor of the Ramond part of the neutral sector develops a cosine term, $T_{\psi}(z)=-\frac{1}{4}(\partial \phi)^{2}-\frac{1}{16 z^{2}} \cos (2 \sqrt{2} \phi)$, a signature of a tunneling phenomenon which selects out the new stable $c=3 / 2$ vacuum; we identify such a theory with the one describing the FFXY model conjectured in Ref. [11].

\section{FFXY phase diagram from TM model}

In this Section we will derive the FFXY phase diagram in terms of the RG flow which originates from perturbing our TM model with relevant operators. We observe that the limit $h \rightarrow 0$ in the Hamiltonian of Eq. (2) gives rise, in the continuum, to a CFT with two scalar boson fields $\varphi^{(i)}$ and with central charge $c=2$. Now a good candidate to describe the FFXY model at criticality around the point $T$ of the phase diagram is a CFT, with $c=2$, which accounts for the full spectrum of excitations of the model: vortices, domain walls, and kinks. The role of the boundary conditions in the description of the excitation spectrum is crucial. In fact, by imposing the coincidence between opposite sides of the square lattice, we obtain a closed geometry, which is the discretized analogue of a torus and gives rise, for the ground state, to two topologically inequivalent configurations, one for even and the other one for odd number of plaquettes. So the ground state on the square lattice maps into the ground state for the even case while it generates two straight domain walls along the two cycles of the torus for the odd case. Such a behaviour has to be taken into account by non trivial boundary conditions on the field $\varphi^{(i)}$ at the borders of the finite lattice. To this aim, let $(-L / 2,0),(L / 2,0)$, $(L / 2, L),(-L / 2, L)$ be the corners of the square lattice $\mathcal{L}$ and assume that the fields $\varphi^{(i)}$ satisfy the boundary conditions $\varphi^{(1)}(r)=\varphi^{(2)}(r)$ for $r \in \mathcal{L} \cap \mathbf{x}$, $\mathbf{x}$ being the $x$ axis. That allows us to consider the two fields $\varphi^{(1)}$ and $\varphi^{(2)}$ on the square lattice $\mathcal{L}$ as the folding of a single field $\mathcal{Q}$, defined on the lattice 
$\mathcal{L}_{0}$ with corners $(-L / 2,-L),(L / 2,-L),(L / 2, L),(-L / 2, L)$. We can implement a discrete version of the 2-reduction procedure by defining the fields $\mathcal{X}(r)=\frac{1}{2}(\mathcal{Q}(r)+\mathcal{Q}(-r)), \Phi(r)=\frac{1}{2}(\mathcal{Q}(r)-\mathcal{Q}(-r))\left(r \in \mathcal{L}_{0}\right)$, which are symmetric and antisymmetric under the action of the group $Z_{2}$. The Hamiltonian (2) can be rewritten in terms of these fields and, for $h=0$, it gives rise, in the continuum, to the TM action $\mathcal{A}=\int\left[\frac{1}{2}(\partial \mathcal{X})^{2}+\frac{1}{2}(\partial \Phi)^{2}\right] d^{2} x$. Let us now show how the phase diagram of the FFXY model can be described by the action:

$$
\mathcal{A}=\int\left[\frac{1}{2}(\partial \mathcal{X})^{2}+\frac{1}{2}(\partial \Phi)^{2}+\mu \cos (\beta \Phi)+\lambda \cos \left(\frac{\beta}{2} \Phi+\delta\right)\right] d^{2} x,
$$

which embodies the higher harmonic potential term conjectured in Refs. [11, $14,7]$. We assume the constraints $\beta^{2}<8 \pi$, which characterizes both the cosine terms as relevant perturbations, and $|\delta| \leq \pi / 2$ [15]. Thus the "neutral" sector is a two-frequency sine-Gordon theory that can be viewed as a deformation of a pure sine-Gordon one with the perturbing term $\lambda \cos (\beta \Phi / 2+\delta)$. The ultraviolet (UV) fixed point $\mu=0, \lambda=0$ of the action (4) corresponds to the TM model with central charge $c=2$, describing the fixed point $T$ in the FFXY phase diagram. In order to study the RG flow in the "neutral" sector let us define the dimensionless variable $\eta \equiv \lambda \mu^{-\left(8 \pi-(\beta / 2)^{2}\right) /\left(8 \pi-\beta^{2}\right)}$; when $\eta=0$ the "neutral" sector reduces to a sine-Gordon model with a particle spectrum built of solitons and antisolitons and, for $\beta^{2}<4 \pi$, some breathers. Switching on the perturbation a confinement of solitons into states with zero topological charge takes place and packets formed by 2 of the original solitons survive as stable excitations for generic values of $|\delta|<\pi / 2$. In the limit $\eta \rightarrow \infty$ the 2 -soliton evolves into the 1 -soliton of the pure sine-Gordon model with $\mu=0$. An unbinding phenomenon takes place in the case $\delta= \pm \pi / 2$ for finite $\eta$ and the 2 -soliton decomposes into a sequence of two kinks $K_{1}$. So the existence of an intermediate critical value $\eta=\eta_{c}$ is required at which a phase transition takes place and the RG flow ends into the infrared (IR) fixed point described by a CFT with central charge $c=1 / 2$, the Ising model. The central charge of the full model (4) so changes from $c=2$ of the UV fixed point to $c=3 / 2$ of the IR fixed point, i.e. we recover early known Monte Carlo results [10]. Such an IR fixed point coincides with the $U(1) \otimes Z_{2}$ symmetric component of the TM model, which results then to properly describe [11] the fixed point $P$ in the phase diagram of the FFXY model. 


\section{References}

[1] G. Cristofano, G. Maiella, V. Marotta, Mod. Phys. Lett. A15 1679 (2000); G. Cristofano, G. Maiella, V. Marotta, G. Niccoli, Nucl. Phys. $B 641547$ (2002).

[2] G. Cristofano, V. Marotta, P. Minnhagen, A. Naddeo, G. Niccoli, J. Stat. Mech. P11009 (2006).

[3] T. C. Halsey, J. Phys. C 182437 (1985); S. Teitel, C. Jayaprakash, Phys. Rev. B 27598 (1983).

[4] J. Lee, Phys. Rev. B 493317 (1994); P. Olsson, Phys. Rev. B 553585 (1997).

[5] J. M. Thijssen, H. J. F. Knops, Phys. Rev. B 422438 (1990); E. Granato, M. P. Nightingale, Phys. Rev. B 487438 (1993).

[6] S. E. Korshunov, Phys. Rev. Lett. 88167007 (2002).

[7] J. M. Thijssen, H. J. F. Knops, Phys. Rev. B 377738 (1988).

[8] P. Olsson, S. Teitel, Phys. Rev. B 71104423 (2005).

[9] E. Granato, J. M. Kosterlitz, Phys. Rev. B 334767 (1986); J. Lee, E. Granato, J. M. Kosterlitz, Phys. Rev. B 444819 (1991).

[10] M. P. Nightingale, E. Granato, J. M. Kosterlitz, Phys. Rev. B 527402 (1995).

[11] O. Foda, Nucl. Phys. B300 611 (1988).

[12] P. Baseilhac, Nucl. Phys. B 636465 (2002).

[13] M. Hasenbusch, A. Pelissetto, E. Vicari, J. Stat. Mech. P12002 (2005).

[14] K. Sokalski, T. Ruijgrok, B. Schoenmaker, Physica A 144322 (1987).

[15] G. Delfino, G. Mussardo, Nucl. Phys. B 516675 (1998). 